\title{
ON ABSOLUTE CONVERGENCE OF MULTIPLE FOURIER SERIES
}

\author{
BY \\ S. MINAKSHISUNDARAM AND OTTO SZÅSZ
}

Introduction. The results of this paper are extensions of corresponding results for simple Fourier series, given by one of the authors (cf. [5])(1). The main problem was to study the relationship between the mean modulus of a function $f(x)$ and series of the type $\sum\left|c_{n}\right| \beta, \beta>0$, where the $c_{n}$ are the Fourier coefficients of $f(x)$. We obtain here analoguous results, employing spherical means of a function of several variables. These means were first used by Bochner [1] in the study of summation of multiple Fourier series.

A particular result is: if $a_{n_{1}} \cdots n_{k}$ are the Fourier coefficients of $f\left(x_{1}, \cdots, x_{k}\right)$, and $f$ satisfies a Lipschitz condition of degree $\alpha$, then $\sum\left|a_{n_{1}} \ldots n_{k}\right| \beta<\infty$ for $\beta>2 \kappa /(\kappa+2 \alpha)$, while the series may be divergent for $\beta=2 \kappa /(\kappa+2 \alpha)$. For some previous results concerning the absolute convergence of double Fourier series cf. [3].

1. Notations. We denote by capital letters vectors in the $k$-dimensional space, so that $X=\left(x_{1}, x_{2}, \cdots, x_{k}\right), N=\left(n_{1}, n_{2}, \cdots, n_{k}\right) ;|N|=\left(\sum_{1}^{k} n_{\nu}^{2}\right)^{1 / 2}$ is the norm of $N ; N X=\sum_{1}^{k} n_{\nu} x_{v}$ is the scalar product of $N$ and $X$. The $x_{1}, \cdots, x_{k}$ are real variables, the $n_{1}, \cdots, n_{k}$ are integers. $f\left(x_{1}, \cdots, x_{k}\right)=f(X)$ is a realvalued integrable function of period $2 \pi$ in each variable. The formal Fourier series of $f(X)$ is

$$
f(X) \sim \sum_{n_{1}} \stackrel{-\infty}{\cdots} \sum_{n_{k}} c_{n_{1}}, \cdots, n_{k} e^{i\left(n_{1} x_{1}+\cdots+n_{k} x_{k}\right)}=\sum c_{N} e^{i N X},
$$

where

$$
c_{N}=\frac{1}{(2 \pi)^{x}} \int_{-\pi}^{\pi} \cdots \int_{-\pi}^{\pi} f(X) e^{-i N X} d X .
$$

$J_{\mu}(x)$ is the Bessel function of order $\mu \geqq 0$ :

we put

$$
J_{\mu}(x)=\sum_{\nu=0}^{\infty}(-1)^{\nu} \frac{(x / 2)^{\mu+2 \nu}}{\nu ! \Gamma(\mu+\nu+1)} ;
$$

$$
\alpha_{\mu}(x)=\frac{2^{\mu} \Gamma(\mu+1) J_{\mu}(x)}{x^{\mu}}=\sum_{\nu=0}^{\infty}(-1)^{\nu} \frac{x^{2 \nu} \Gamma(\mu+1)}{4^{\nu} \nu ! \Gamma(\mu+\nu+1)}
$$

Presented to the Society, April 27, 1946; received by the editors April 15, 1946.

(1) Numbers in brackets refer to the bibliography at the end of the paper. 


$$
A_{n}(X)=\sum_{|N|^{2}=n} c_{N} \exp (i N X), \quad \text { so that } f(X) \sim \sum_{n=0}^{\infty} A_{n}(X) .
$$

We shall denote by $\omega(t)$ a positive function of $t$, decreasing to zero as $t \downarrow 0$

2. Lemmas. We give here some auxiliary theorems.

LEMMA 1. If $R_{k}(n)$ is the number of lattice points in the sphere $\sum_{1}^{n} x_{v}^{2} \leqq n$, then

$$
R_{k}(n)=O\left(n^{\kappa / 2}\right) \equiv O n^{\kappa / 2}
$$

as $n \rightarrow \infty$.

Actually the sharper estimate is known [cf. 2, p. 825]:

$$
R_{\kappa}(n)=\frac{\pi^{\kappa / 2} n^{\kappa / 2}}{\Gamma(1+\kappa / 2)}+O n^{\kappa(\kappa-1) / 2(\kappa+1)}
$$

LEMMA 2. For $\mu \geqq 0$, $x$ real or complex,

$$
\begin{aligned}
\alpha_{\mu}(x) & =\frac{2 \Gamma(\mu+1)}{\Gamma(\mu+1 / 2) \Gamma(1 / 2)} \int_{0}^{\pi / 2} \cos (x \cos t) \sin ^{2 \mu} t d t \\
& =\frac{\Gamma(\mu+1)}{\Gamma(\mu+1 / 2) \Gamma(1 / 2)} \int_{0}^{\pi} e^{i x \cos t} \sin ^{2 \mu} t d t .
\end{aligned}
$$

The proof follows on using the cosine series or exponential series and integrating termwise [6, pp. 47-48].

Corollary. For real $x$

$$
\left|\alpha_{\mu}(x)\right| \leqq \frac{2 \Gamma(\mu+1)}{\Gamma(\mu+1 / 2) \Gamma(1 / 2)} \int_{0}^{\pi / 2} \sin ^{2 \mu} t d t=\alpha_{\mu}(0)=1 .
$$

For $\mu=0,(2.3)$ reduces to $\left|J_{0}(x)\right| \leqq 1$, an inequality given by Hansen [6, p. 31].

LEMMA 3. For any $u>0$ and $a$ corresponding constant $b(u)>0, b(u)<1$ $-\alpha_{\mu}(x)<2$ for $x>u$; moreover

and

$$
1-\alpha_{\mu}(x)>\frac{x^{2}}{\pi^{2}(\mu+1)} \quad \text { for } 0<x<\pi,
$$

$$
1-\alpha_{\mu}(x)<(x / 2)^{2} \frac{1}{\mu+1} \quad \text { for } x>0 .
$$

Proof. From (2.2) and (2.3), putting $2 \Gamma(\mu+1) / \Gamma(\mu+1 / 2) \Gamma(1 / 2)=\gamma(\mu)$, we have

$$
1-\alpha_{\mu}(x)=\gamma(\mu) \int_{0}^{\pi / 2}\{1-\cos (x \cos t)\} \sin ^{2 \mu} t d t>0 \quad \text { for } x>0 .
$$


It is known that $J_{\mu}(x) \rightarrow 0$ as $x \rightarrow \infty$, hence $\alpha_{\mu}(x) \rightarrow 0$; thus for some $b(u)>0$

$$
1-\alpha_{\mu}(x)>b(u) \quad \text { for } x>u \text {. }
$$

Furthermore from (2.4) and (2.3)

$$
1-\alpha_{\mu}(x)<2 \gamma(\mu) \int_{0}^{\pi / 2} \sin ^{2 \mu} t d t=2, \quad \text { for } x>0 .
$$

Finally, for $0<x<\pi$,

$$
1-\cos (x \cos t)=2 \sin ^{2}\left(\frac{x}{2} \cos t\right)\left\{\begin{array}{l}
>\frac{2 x^{2}}{\pi^{2}} \cos ^{2} t \\
<\frac{x^{2}}{2} \cos ^{2} t
\end{array}\right.
$$

hence

$$
\begin{aligned}
1-\alpha_{\mu}(x)>\frac{2 \gamma(\mu)}{\pi^{2}} x^{2} \int_{0}^{\pi / 2} \cos ^{2} t \sin ^{2 \mu} t d t & =\frac{2 \gamma(\mu)}{\pi^{2}} x^{2}\left\{\frac{1}{\gamma(\mu)}-\frac{1}{\gamma(\mu+1)}\right\} \\
& =\frac{x^{2}}{\pi^{2}(\mu+1)},
\end{aligned}
$$

and

$$
1-\alpha_{\mu}(x)<\frac{\gamma(\mu) x^{2}}{2} \int_{0}^{\pi / 2} \cos ^{2} t \sin ^{2 \mu} t d t=\frac{x^{2}}{4(\mu+1)} ;
$$

this proves the lemma.

LEMMA 4. Let $h$ be real, $r>0, \delta>0$, then the following statements are equivalent:

$$
\begin{gathered}
\sum_{n=1}^{\infty} n^{r h-1} \omega\left(\delta n^{-r}\right)<\infty, \\
\sum_{\lambda=1}^{\infty} 2^{\lambda r h} \omega\left(\delta \cdot 2^{-\lambda r}\right)<\infty, \\
\int_{1}^{\infty} t^{h-1} \omega\left(\frac{1}{t}\right) d t<\infty .
\end{gathered}
$$

Proof. We have for $r h \geqq 1$

$$
2^{(\lambda-1) r h} \omega\left(\delta \cdot 2^{-\lambda r}\right)<\sum_{\nu=2 \lambda-1}^{2^{\lambda}-1} \nu^{r h-1} \omega\left(\delta \cdot \nu^{-r}\right)<2^{\lambda+h} \omega\left(\delta \cdot 2^{-(\lambda-1) r}\right),
$$

hence

$$
2^{-r h} \sum_{\lambda=1}^{\infty} 2^{\lambda r h} \omega\left(\delta \cdot 2^{-\lambda r}\right)<\sum_{n=1}^{\infty} n^{r h-1} \omega\left(\delta \cdot n^{-r}\right)<2^{r h} \sum_{0}^{\infty} 2^{\lambda r h} \omega\left(\delta \cdot 2^{-\lambda r}\right),
$$


with similar inequalities for $r h<1$; hence $(2.5)$ and $\left(2.5^{\prime}\right)$ are equivalent. We also have for $r h \leqq 1$

$$
\int_{n}^{n+1} x^{r h-1} \omega\left(\delta x^{-r}\right) d x<n^{r h-1} \omega\left(\delta n^{-r}\right)<\int_{n-1}^{n} x^{r h-1} \omega\left(\delta x^{-r}\right) d x
$$

hence

$$
\int_{1}^{\infty} x^{r h-1} \omega\left(\delta x^{-r}\right) d x<\sum_{1}^{\infty} n^{r h-1} \omega\left(\delta n^{-r}\right)<\int_{0}^{\infty} x^{r h-1} \omega\left(\delta x^{-r}\right) d x,
$$

with similar inequalities for $r h>1$; the substitution $x^{r}=\delta t$ yields the equivalence of (2.5) and (2.6). This proves the lemma.

In view of (2.6), $r$ and $\delta$ are not necessarily the same in the different statements.

COROLLARY. The following statements are equivalent:

$$
\sum n^{(n / k)-1} \omega\left(\delta n^{-1 / k}\right)<\infty
$$

and

$$
\sum 2^{\lambda h / 2} \omega\left(\delta \cdot 2^{-\lambda / 2}\right)<\infty .
$$

This follows on putting $r=1 / \kappa$ in (2.5), and $r=1 / 2$ in $\left(2.5^{\prime}\right)$.

LEMMA 5. If $a_{\nu} \geqq 0$, and $r>0$, then the two statements are equivalent:

$$
\sum_{1}^{\infty} a_{\nu}\left|1-\alpha_{\mu}\left(t \nu^{1 / 2}\right)\right| r=O \omega(t)
$$

and

$$
n^{-r} \sum_{1}^{n} \nu^{r} a_{\nu}+\sum_{n+1}^{\infty} a_{r}=O \omega\left(\delta n^{-1 / 2}\right) \quad \text { as } n \rightarrow \infty,
$$

$\delta$ being an arbitrary positive number.

Assume first that (2.8) holds; given $t>0$ choose

$$
n=\left[\delta^{2} t^{2}\right] \leqq \delta^{2} t^{2}<n+1
$$

then from Lemma 3

$$
\sum_{1}^{n+1} a_{\nu}\left|1-\alpha_{\mu}\left(t \nu^{1 / 2}\right)\right| r<\frac{t^{2 r}}{4^{r}(\mu+1)^{r}} \sum_{1}^{n+1} \nu^{r} a_{\nu}=O t^{2 r} n^{r} \omega\left(\delta(n+1)^{-1 / 2}\right)=O \omega(t),
$$

and

$$
\sum_{n+2}^{\infty} a_{\nu}\left|1-\alpha_{\mu}\left(t \nu^{1 / 2}\right)\right| r<2 \sum_{n+2}^{\infty} a_{\nu}=O \omega\left(\delta(n+1)^{-1 / 2}\right)=O \omega(t) .
$$

Conversely, if (2.7) holds, choose for a given $n$ and $\delta>0$ 


$$
t=\min \left(\pi n^{-1 / 2}, \delta n^{-1 / 2}\right),
$$

then

$$
\sum_{1}^{n} a_{\nu}\left|1-\alpha_{\mu}\left(t \nu^{1 / 2}\right)\right| r>\frac{t^{2 r}}{\pi^{2 r}(\mu+1)^{r}} \sum_{1}^{n} \nu^{r} a_{\nu}
$$

hence

$$
n^{-r} \sum_{1}^{n} \nu^{p} a_{\nu}=O \omega(t)=O \omega\left(\delta n^{-1 / 2}\right) .
$$

Furthermore, using again Lemma 3, we have

hence

$$
\sum_{n+1}^{\infty} a_{\nu}\left|1-\alpha_{\mu}\left(t \nu^{1 / 2}\right)\right| r>b \sum_{n+1}^{\infty} a_{\nu} \quad(b \text { a constant }),
$$

$$
\sum_{n+1}^{\infty} a_{\nu}=O \omega(t)=O \omega\left(\delta n^{-1 / 2}\right) .
$$

This proves the lemma. It follows that if $(2.8)$ holds for some $\delta>0$, it holds for any $\delta>0$.

Lemma 6. Assume that for some $\delta>0$

$$
\sum_{\lambda=1}^{\infty} \omega\left(\delta 2^{-\lambda} n^{-1 / 2}\right)=O \omega\left(\delta n^{-1 / 2}\right)
$$

as $n \rightarrow \infty$,

and let $r>0, a_{\nu} \geqq 0$; then the following statements are equivalent:

$$
\begin{array}{rlrl}
n^{-r} \sum_{1}^{n} \nu^{r} a_{\nu}+\sum_{n+1}^{\infty} a_{\nu} & =O \omega\left(\delta n^{-1 / 2}\right), & n \rightarrow \infty, \\
n^{-r} \sum_{1}^{n} \nu^{r} a_{\nu} & =O \omega\left(\delta n^{-1 / 2}\right), & & \\
\sum_{1}^{\infty} a_{\nu}\left|1-\alpha_{\mu}\left(t \nu^{1 / 2}\right)\right| r & =O \omega(t), & t \rightarrow 0 .
\end{array}
$$

The equivalence of (2.10) and (2.11) follows from Lemma (2.5) in [5]; the equivalence of (2.11) and (2.12) follows from Lemma 5. This proves Lemma 6.

LEMMA 7. Young-Hausdorff inequality. If $1<p \leqq 2$, and

$$
f(X) \sim \sum c_{N} \exp (i N X),
$$

then

$$
\left\{\sum\left|c_{N}\right|^{p^{\prime}}\right\}^{1 / p^{\prime}} \leqq M_{p}(f) \equiv M_{p} f
$$

and 


$$
M_{p}^{p} f \leqq \sum\left|c_{N}\right|^{p}
$$

where $1 / p+1 / p^{\prime}=1$, and

(cf. [4]).

$$
M_{p}^{p} f=\frac{1}{(2 \pi)^{x}} \int_{-\pi}^{\pi}|f(X)|^{p} d X
$$

Denote by $f(X ; t)$ the spherical mean of $f(X)$ over the surface of the sphere of radius $t$ and center $x$; then $[1$, p. 177]

$$
\begin{aligned}
f(X ; t)= & (2 \pi)^{-\kappa / 2} \Gamma\left(\frac{\kappa}{2}\right) \int_{\sigma} f\left(x_{1}+t \xi_{1}, \cdots, x_{\kappa}+t \xi_{\kappa}\right) d \sigma_{\xi} \\
& \sim \sum_{N} c_{N} \alpha_{\mu}(t|N|) \exp (i N X) \\
& \sim \sum_{n=0}^{\infty} \alpha_{\mu}\left(t n^{1 / 2}\right) A_{n}(x), \quad \mu=(\kappa-2) / 2 ;
\end{aligned}
$$

$\sigma$ denotes the unit sphere $\xi_{1}^{2}+\cdots+\xi_{\kappa}^{2}=1, d \sigma_{\xi}$ its $(\kappa-1)$-dimensional volume element. Thus, putting $f(X ; t)-f(X)=\phi(X ; t)$, we have

$$
\begin{aligned}
\phi(X ; t) & \backsim \sum_{n} c_{N}\left\{\alpha_{\mu}(t|N|)-1\right\} \exp (i N X) \\
& \sim \sum_{n=0}^{\infty}\left\{\alpha_{\mu}\left(t n^{1 / 2}\right)-1\right\} A_{n}(x) .
\end{aligned}
$$

LEMMA 8. If $M_{1} \phi(X ; t)=O \omega(t)$ as $t \rightarrow 0$, then for any $\delta>0$

$$
c_{N}=O \omega\left(\frac{\delta}{|N|}\right)
$$

as $|N| \rightarrow \infty$.

It follows from (1.1), (1.2) and (2.14) that

$$
c_{N}\left\{\alpha_{\mu}(t|N|)-1\right\}=(2 \pi)^{-\kappa} \Gamma\left(\frac{\kappa}{2}\right) \int_{-\pi}^{\pi} \phi(X ; t) \exp (-i N X) d X,
$$

hence

$$
\left|c_{N}\right|\left|1-\alpha_{\mu}(t|N|)\right| \leqq M_{1} \phi(X ; t)=O \omega(t) .
$$

Lemma 8 now follows from Lemma 3 , on putting $t|N|=\delta$.

LEMMA 9. Let $P_{n}(z)=\sum_{0}^{n} c_{\nu} z^{p}, 1 \leqq p \leqq \infty ;$ if

$$
M_{p} P_{n}(z) \leqq 1
$$$$
\text { for }|z| \leqq 1 \text {, }
$$

then

$$
M_{p} P_{n}^{\prime}(z) \leqq n
$$

(cf. $[5$, p. 385]).

$$
\text { Note. For } p=\infty, M_{p} P(z)=\max |P(z)| \text { for }|z| \leqq 1 \text {. }
$$


We shall frequently use Hölder's and Minkowski's well known inequalities for multiple series and integrals (cf. Hardy, Littlewood, and Pólya, Inequalities, Cambridge, 1934).

3. A theorem on absolute convergence. We now present our main criterion for absolute convergence.

THEOREM 1. If, with the notations of $\S 2,1 \leqq p \leqq 2, f(X) \in L_{p}$,

$$
M \phi(X ; t)=O \omega(t)
$$

as $t \rightarrow 0$,

and

$$
\sum_{1}^{\infty} n^{-\beta / p^{\prime}} \omega^{\beta}\left(\delta n^{-1 / x}\right)<\infty
$$

then

$$
\sum\left|c_{N}\right|^{\beta}<\infty \text {. }
$$

By (3.1) and Lemma 7 for $1<p \leqq 2, \sum\left|c_{N}\right|^{p^{\prime}}\left|1-\alpha_{\mu}(t|N|)\right|^{p^{\prime}}=O \omega^{p^{\prime}}(t)$, or

$$
\sum_{1}^{\infty} \rho_{n}^{p^{\prime}}\left|1-\alpha_{\mu}\left(t n^{1 / 2}\right)\right|^{p^{\prime}}=O \omega^{p^{\prime}}(t)
$$

where $\rho_{n}=\rho_{n}(p)$ is defined by

$$
\rho_{n}^{p^{\prime}}=\sum_{|N|^{2}-n}\left|c_{N}\right|^{p^{\prime}}=\sum\left|c_{n_{1}} \cdots n_{k}\right|^{p^{\prime}} \quad\left(n_{1}^{2}+\cdots+n_{x}^{2}=n\right) .
$$

By Lemma 5, (3.4) is equivalent to

hence

$$
n^{-p^{\prime}} \sum_{1}^{n} \nu^{p^{\prime}} \rho_{\nu}^{p^{\prime}}+\sum_{n+1}^{\infty} \rho_{\nu}^{p^{\prime}}=O \omega^{p^{\prime}}\left(\delta n^{-1 / 2}\right),
$$

$$
\sum_{n+1}^{2 n} \rho_{\nu}^{p^{\prime}}=O \omega^{p^{\prime}}\left(\delta n^{-1 / 2}\right)
$$

By the Hölder inequality for $q>1,1 / q+1 / q^{\prime}=1$,

$$
\sum_{n}^{2 n} \rho_{\nu}^{\beta}=\sum_{n \leqq|N|^{2} \leq 2 n}\left|c_{N}\right|^{\beta} \leqq\left(\sum\left|c_{N}\right|^{\beta q}\right)^{1 / q}\left(\sum 1\right)^{1 / q^{\prime}} ;
$$

let first $\beta<p^{\prime}$; choose

$$
\beta q=p^{\prime}, \text { hence } q^{\prime}=\frac{q}{q-1}=\frac{p^{\prime}}{p^{\prime}-\beta} .
$$

Now, from (3.5) and (2.1) 


$$
\sum_{n}^{2 n} \rho_{\nu}^{\beta}=O \omega^{\beta}\left(\delta n^{-1 / 2}\right)\left(R_{k}(2 n)\right)^{1-\beta / p^{\prime}}=O n^{\left(1-\beta / p^{\prime}\right) \kappa / 2} \omega^{\beta}\left(\delta n^{-1 / 2}\right)
$$

Putting $n=2^{\lambda}, \lambda=0,1, \cdots$, and summing over $\lambda$ yields

$$
\sum_{1}^{\infty} \rho_{\nu}^{\beta}=0 \sum_{\lambda=0}^{\infty} 2^{\lambda \kappa\left(1-\beta / p^{\prime}\right) / 2} \omega^{\beta}\left(\delta 2^{-\lambda / 2}\right)
$$

the right side is convergent by the corollary to Lemma 4 (with $h=\kappa\left(1-\beta / p^{\prime}\right)$ ) and by (3.2). Hence (3.3) holds.

Next if $\beta=p^{\prime}>1$, then (3.3) follows from (2.13), if we assume only, instead of (3.1), that $f(X) \in L_{p}$; a fortiori

$$
\sum\left|c_{N}\right|^{\beta}<\infty
$$

for $\beta \geqq p^{\prime}$.

Finally let $p=1 ;(3.2)$ becomes

$$
\sum \omega^{\beta}\left(\delta n^{-1 / x}\right)<\infty .
$$

Denote by $r_{k}(n)$ the number of lattice points on the circle $\sum_{1}^{\kappa} x_{\nu}^{2}=n$; thus $\sum_{0}^{n} r_{k}(\nu)=R_{k}(n)$.

From Lemma 8, for any $\delta>0$

$$
\sum_{|N|^{2}=n}\left|c_{N}\right|^{\beta}=O \sum \omega^{\beta}\left(\delta|N|^{-1}\right)=O r_{k}(n) \omega^{\beta}\left(\delta n^{-1 / 2}\right) ;
$$

furthermore from (3.6) and Lemma 4 (with $h=\kappa$ )

$$
\sum_{1}^{\infty} n^{\kappa / 2-1} \omega^{\beta}\left(\delta n^{-1 / 2}\right)<\infty \text {. }
$$

Now, using (2.1), we have

$$
\begin{aligned}
\sum_{1}^{n} r_{\kappa}(\nu) \omega^{\beta}\left(\delta \nu^{-1 / 2}\right) & =\sum_{1}^{n} R_{\kappa}(\nu) \omega^{\beta}\left(\delta \nu^{-1 / 2}\right)-\sum_{0}^{n-1} R_{k}(\nu) \omega^{\beta}\left(\delta(\nu+1)^{-1 / 2}\right) \\
& \leqq R_{\kappa}(n) \omega^{\beta}\left(\delta n^{-1 / 2}\right)+\sum_{1}^{n-1} R_{\kappa}(\nu)\left\{\omega^{\beta}\left(\delta \nu^{-1 / 2}\right)-\omega^{\beta}\left(\delta(\nu+1)^{-1 / 2}\right)\right\} \\
& =O n^{\alpha / 2} \omega^{\beta}\left(\delta n^{-1 / 2}\right)+O \sum_{1}^{n-1} \nu^{\kappa / 2}\left\{\omega^{\beta}\left(\delta \nu^{-1 / 2}\right)-\omega^{\beta}\left(\delta(\nu+1)^{-1 / 2}\right)\right\} \\
& =O \sum_{1}^{n}\left\{\nu^{\alpha / 2}-(\nu-1)^{\kappa / 2}\right\} \omega^{\beta}\left(\delta \nu^{-1 / 2}\right) \\
& =O(1), \quad \text { as } n \rightarrow \infty .
\end{aligned}
$$

This completes the proof of Theorem 1 . 
Actually we can prove for $\beta=p^{\prime}$ that

$$
\sum \rho_{n}^{p^{\prime}} \log n<\infty \text {. }
$$

4. Converse theorems. We give here two theorems to be employed in subsequent sections.

TheOREM 2. Let $1 \leqq p \leqq 2 ;$ assume that

$$
\sum_{\lambda=1}^{\infty} \omega^{p}\left(\delta 2^{-\lambda} n^{-1 / 2}\right)=O \omega^{p}\left(\delta n^{-1 / 2}\right), \quad \quad \text { as } n \rightarrow \infty,
$$

and that

$$
\sum_{1}^{n} \nu^{p} \rho_{\nu}^{p}=O n^{p} \omega^{p}\left(\delta n^{-1 / 2}\right)
$$

as $n \rightarrow \infty$;

then

$$
M_{p^{\prime}} \phi(X ; t)=O \omega(t),
$$

as $t \rightarrow 0$.

Note. If $p=1, p^{\prime}=\infty$, then $M_{p^{\prime}}$ means the effective upper bound of $|\phi(X ; t)|$ in the region of $X$.

Proof. By Lemma 6, (4.2) is equivalent to

that is,

$$
\sum_{1}^{\infty} \rho_{n}^{p}\left|1-\alpha_{\mu}\left(t n^{1 / 2}\right)\right|^{p}=O \omega^{p}(t)
$$

$$
\sum\left|c_{N}\right|^{p}\left|1-\alpha_{\mu}(t|N|)\right|^{p}=O \omega^{p}(t) .
$$

Now from (2.14) and Lemma 7 (which holds also for $p=1$ )

$$
M_{p}, \phi(X ; t)=O \omega(t)
$$

this proves the theorem.

Note that (4.2) means:

$$
\sum_{|N|^{2} \leq n}|N| p\left|c_{N}\right|^{p}=O n^{p} \omega^{p}\left(\delta n^{-1 / 2}\right) .
$$

TheOREM 3. Assume that $\omega(t) \downarrow 0$ as $t \downarrow 0$, and that

$$
\sum_{\lambda=1}^{\infty} \omega^{2}\left(2^{-\lambda} \delta n^{-1 / 2}\right)=O \omega^{2}\left(\delta n^{-1 / 2}\right) \quad \text { as } n \rightarrow \infty .
$$

Then a necessary and sufficient condition that

$$
M_{2} \phi(X ; t)=O \omega(t)
$$

as $t \rightarrow 0$,

is that 


$$
\sum_{1}^{n} \nu^{2} \rho_{\nu}^{2}=O n^{2} \omega^{2}\left(\delta n^{-1 / 2}\right)
$$

as $n \rightarrow \infty$.

First if (4.4) holds then (4.3) follows by Theorem 2 (for $p=2$ ). Conversely if (4.3) holds, then from (2.14) and Lemma 7

$$
\sum\left|c_{N}\right|^{2}\left|1-\alpha_{\mu}(t|N|)\right|^{2}=O \omega^{2}(t),
$$

which by Lemma 6 is equivalent to (4.4).

5. Counter examples. For $\beta=1$, Theorem 1 becomes:

THEOREM $1^{\prime}$. If $M_{p} \phi(t)=O \omega(t)$ as $t \rightarrow 0$, and

$$
\sum n^{-1 / p^{\prime}} \omega\left(\delta n^{-1 / \kappa}\right)<\infty,
$$

then

$$
\sum\left|c_{N}\right|<\infty .
$$

To show that this result is the best possible we shall prove:

THEOREM 4. Let $\omega(t)$, in addition to having the property $\omega(t) \downarrow 0$ as $t \downarrow 0$, be such that

$$
\int_{1}^{u} \omega\left(t^{-1}\right) d t=O u \omega\left(u^{-1}\right) \quad \text { as } u \rightarrow \infty \text {, }
$$

while

$$
\sum n^{-1 / p^{\prime} \omega\left(\delta n^{-1 / x}\right)=\infty,}
$$

where $1 \leqq p \leqq 2$.

Then there exists a function $f(X) \in L_{p}$, such that

$$
M_{p} \phi(X ; t)=O \omega(t),
$$

while

$$
\sum\left|c_{N}\right|=\infty \text {. }
$$

By Lemma 4 and its corollary (with $h=\kappa / p$ ) (5.1) is equivalent to

$$
\sum_{\lambda=1}^{\infty} 2^{\alpha \lambda / p} \omega\left(\delta 2^{-\lambda}\right)<\infty,
$$

while (5.3) is equivalent to

$$
\sum 2^{\kappa \lambda / p} \omega\left(\delta 2^{-\lambda}\right)=\infty \text {. }
$$

We define $\epsilon_{n}=\omega\left(2^{-n} \delta\right), \lambda_{n}=2^{n+1}+n-2$,

$$
g_{n}(z)=2^{-n\left(1+1 / p^{\prime}\right)}\left(\sum_{0}^{2^{n}} z^{\nu}\right)^{2}, \quad n=0,1,2, \ldots ;
$$


so that

$$
\lambda_{n+1}-\lambda_{n}=2^{n+1}+1
$$

Construct the power series

$$
G(Z)=\sum_{n=0}^{\infty}\left(\epsilon_{n}-\epsilon_{n+1}\right)\left(z_{1} \cdots z_{k}\right)^{\lambda} \prod_{n=1}^{k} g_{n}\left(z_{n}\right) ;
$$

then $G(Z)$ has the formal power series

$$
G(Z)=\sum_{n_{1}=0}^{\infty} \cdots \sum_{n_{n}=0}^{\infty} \gamma_{n_{1}} \cdots n_{n} z_{1}^{n_{1}} \cdots z_{x}^{n_{x}} .
$$

It is clear from the construction that $\gamma_{N} \geqq 0$; putting $Z=1$ we find

$$
\begin{aligned}
\sum \gamma_{N} & >\sum_{n=0}^{m}\left(\epsilon_{n}-\epsilon_{n+1}\right) 2^{-\kappa n\left(1+1 / p^{\prime}\right)}\left(2^{n+1}-1\right)^{2 \kappa}>\sum_{0}^{m}\left(\epsilon_{n}-\epsilon_{n+1}\right) 2^{\kappa n / p} \\
& >\sum_{1}^{m}\left(\epsilon_{n}-\epsilon_{m+1}\right) 2^{\kappa n / p}\left(1-2^{-\kappa / p}\right) .
\end{aligned}
$$

For a given integer $l$ choose $m$ so large that $\epsilon_{l}>2 \epsilon_{m+1}$, then

$$
\sum \gamma_{N}>\frac{1}{2}\left(1-2^{-\alpha / p}\right) \sum_{1}^{l} \epsilon_{n} 2^{\kappa n / p} \rightarrow \infty \quad \text { as } l \rightarrow \infty,
$$

by $\left(5.3^{\prime}\right)$. Hence

$$
\sum \gamma_{N}=\infty \text {. }
$$

We next show that for $z_{\nu}=e^{i x_{\nu}}, \nu=1,2, \cdots, \kappa, G(Z)$ becomes the Fourier power series of a function $F(X) \in L_{p}$. Write

$$
u_{n}(Z)=\left(\epsilon_{n}-\epsilon_{n+1}\right)\left(z_{1} \cdots z_{k}\right)^{\lambda_{n}} \prod_{n=1}^{\kappa} g_{n}\left(z_{n}\right),
$$

then for $z_{p}=e^{i x_{p}}$

$$
\begin{aligned}
M_{p} u_{n} & =\left(\epsilon_{n}-\epsilon_{n+1}\right) \frac{1}{(2 \pi)^{\kappa}} 2^{-\kappa n\left(1+1 / p^{\prime}\right)}\left(\int_{-\pi}^{\pi}\left|\sum_{0}^{2^{n}} e^{i \nu x}\right| 2 p d x\right)^{\kappa / p} \\
& =O\left(\epsilon_{n}-\epsilon_{n+1}\right) 2^{-\kappa n\left(1+1 / p^{\prime}\right)}\left(\int_{0}^{\pi}\left|\frac{\sin \left(2^{n}+1\right) x / 2}{x}\right|^{2 p} d x\right)^{\kappa / p} \\
& =O\left(\epsilon_{n}-\epsilon_{n+1}\right) 2^{-\kappa n\left(1+1 / p^{\prime}\right)} 2^{n(2 p-1) \times / p}=O\left(\epsilon_{n}-\epsilon_{n+1}\right)
\end{aligned}
$$

hence, by Minkowski's inequality,

$$
M_{p} G \leqq \sum_{n=0}^{\infty} M_{p} u_{n}=O(1) .
$$


We shall finally prove (5.4); we have

$$
F(X ; t)-F(X)=\sum_{\nu=0}^{\infty}\left\{u_{\nu}(X ; t)-u_{\nu}(X)\right\},
$$

hence $M_{p} \phi \leqq \sum_{\nu=0}^{\infty} M_{p}\left\{u_{\nu}(X ; t)-u_{\nu}(X)\right\}=\sum_{\nu=0}^{n}+\sum_{n+1}^{\infty}=S_{1}+S_{2}$, say. Now, by Minkowski's inequality and (2.14),

$$
\begin{aligned}
M_{p} u_{v}(X ; t) & =\Gamma\left(\frac{\kappa}{2}\right) 2^{-\kappa-1} \pi^{3 \kappa / 2}\left(\int_{-\pi}^{\pi}\left|\int_{\sigma} u_{v}\left(x_{1}+t \xi_{1}, \cdots, x_{\kappa}+t \xi_{\kappa}\right) d \sigma_{\xi}\right|^{p} d X\right)^{1 / p} \\
& \leqq \Gamma\left(\frac{\kappa}{2}\right) 2^{-\kappa-1} \pi^{3 \kappa / 2} \int_{\sigma}\left(\int_{-\pi}^{\pi}\left|u_{v}\left(x_{1}+t \xi_{1}, \cdots, x_{\kappa}+t \xi_{k}\right)\right|^{p} d X\right)^{1 / p} d \sigma_{\xi} \\
& =2^{-1} \Gamma\left(\frac{\kappa}{2}\right) \pi^{-\alpha / 2} \int_{\sigma} M_{p}\left(u_{v}\right) d \sigma_{\xi}
\end{aligned}
$$

hence, if we use (5.7), $S_{2}=O \epsilon_{n}$. Furthermore

$$
M_{p}\left\{u_{\nu}(X ; t)-u_{\nu}(X)\right\} \leqq 2^{-1} \Gamma\left(\frac{\kappa}{2}\right) \pi^{-x / 2} \int_{0} M_{p}\left\{u_{\nu}(X ; t)-u_{\nu}(X)\right\} d \sigma_{\xi} ;
$$

from the mean value theorem

$$
u_{\nu}(X ; t)-u_{\nu}(X)=t \sum_{\lambda=1}^{k} \xi_{\lambda} \frac{\partial u_{\nu}}{\partial x_{\lambda}}(X ; \theta t), \quad \text { where } 0<\theta<1 ;
$$

hence from Minkowski's inequality

$$
\begin{aligned}
M_{p}\left\{u_{\nu}(X ; t)-u_{\nu}(X)\right\} & \leqq t \sum_{\lambda=1}^{\kappa}\left|\xi_{\lambda}\right| M_{p} \frac{\partial u_{\nu}}{\partial x_{\lambda}}(X ; \theta t) \\
& \leqq(2 \pi)^{-\kappa / 2 t} \int_{\sigma} M_{p} \frac{\partial u_{\nu}}{\partial x_{\lambda}}(X) d \sigma_{\xi_{0}}
\end{aligned}
$$

We now employ Lemma 9; thus from (5.5) and (5.6)

$$
M_{p}\left\{u_{\nu}(X ; t)-u_{\nu}(X)\right\}=t O\left(\epsilon_{\nu}-\epsilon_{\nu+1}\right)\left(2^{\nu+1}+\lambda_{\nu}\right)=t O 2^{\nu}\left(\epsilon_{\nu}-\epsilon_{\nu+1}\right) .
$$

It follows that

$$
\begin{aligned}
S_{1} & =t O \sum_{0}^{n} 2^{\nu}\left(\epsilon_{\nu}-\epsilon_{\nu+1}\right)=O t \sum_{0}^{n}\left(2^{\nu+1}-2^{\nu}\right) \epsilon_{\nu} \\
& =O t \sum_{0}^{n}\left(2^{\nu+1}-2^{\nu}\right) \omega\left(\delta 2^{-\nu}\right)=O t \int_{0}^{2^{n}} \omega\left(\delta x^{-1}\right) d x \\
& =O t 2^{n} \omega\left(\delta 2^{-n}\right),
\end{aligned}
$$


by (5.2). We now choose $n$ so that for a given positive $t<\delta$

$$
2^{n-1}<\delta t^{-1} \leqq 2^{n},
$$

then

$$
S_{1}=O \omega(t) \text {, and } S_{2}=O \omega(t),
$$

and the proof of Theorem 4 is complete.

A simpler example, but of a special type, is

$$
G(Z)=\sum_{n=0}^{\infty}\left(\epsilon_{n}-\epsilon_{n+1}\right) \sum_{n=1}^{k} z_{\eta}^{\lambda_{n}} g_{n}\left(z_{n}\right) .
$$

6. The case $p=2$ and arbitrary $\beta>0$. For the case $p=2$, Theorem 1 becomes:

THEOREM. $1^{\prime \prime}$. If $M_{2} \phi(t)=O \omega(t)$, and for some $\beta>0$

then

$$
\sum n^{-\beta / 2} \omega^{\beta}\left(\delta n^{-1 / k}\right)<\infty,
$$

$$
\sum\left|c_{N}\right|^{\beta}<\infty \text {. }
$$

We now prove:

THEOREM 5. Let $\omega(t)$, in addition to having the property $\omega(t) \downarrow 0$ as $t \downarrow 0$, be such that

$$
\int_{1}^{u} x \omega^{2}\left(x^{-1}\right) d x=O u^{2} \omega^{2}\left(u^{-1}\right) \quad \text { as } u \rightarrow \infty,
$$

while for a given positive $\beta<2$

$$
\sum n^{-\beta / 2} \omega^{\beta}\left(\delta n^{-1 / \alpha}\right)=\infty .
$$

Then there exists a function $f(X) \in L_{2}$, such that

$$
M_{2} \phi(X ; t)=O \omega(t), \quad t \rightarrow 0,
$$

but

$$
\sum\left|c_{N}\right|^{\beta}=\infty .
$$

We employ again the polynomial (5.5), where now $p^{\prime}=2$, and the polynomial (5.6), replacing the factor $\epsilon_{n}-\epsilon_{n+1}$ by

$$
\left(\epsilon_{n}^{\beta}-\epsilon_{n+1}^{\beta}\right)^{1 / \beta}=\alpha_{n},
$$

say.

As before $\epsilon_{n}=\omega\left(\delta 2^{-n}\right)$. On writing

$$
G(Z)=\sum_{n=0}^{\infty} u_{n}(Z)=\sum \gamma_{N} z_{1}^{n_{1}} \cdots z_{k}^{n_{k}},
$$


we have again $\gamma_{N} \geqq 0$. Now

$$
\begin{aligned}
\sum \gamma_{N}^{\beta} & >\sum \alpha_{n}^{\beta} 2^{-3 n \beta \kappa / 2}\left(\sum_{1}^{2^{n}} \nu^{\beta}\right) \\
& >\frac{1}{(\beta+1)^{\kappa}} \sum_{n=0}^{m} \alpha_{n}^{\beta} 2^{n \kappa(1-\beta / 2)}=\frac{1}{(\beta+1)^{\kappa}} \sum_{0}^{m}\left(\epsilon_{n}^{\beta}-\epsilon_{n+1}^{\beta}\right) 2^{n \kappa(1-\beta / 2)} \\
& >\left(2^{\kappa(1-\beta / 2)}-1\right) \sum_{1}^{m}\left(\epsilon_{n}^{\beta}-\epsilon_{m+1}^{\beta}\right) 2^{(n-1)(1-\beta / 2) \kappa} .
\end{aligned}
$$

Hence for $\epsilon_{l}^{\beta}>2 \epsilon_{m+1}^{\beta}$

$$
\sum \gamma_{N}^{\beta}>\frac{1}{2}\left(2^{\kappa(1-\beta / 2)}-1\right) \sum_{1}^{l} \epsilon_{n}^{\beta} 2^{\alpha(n-1)(1-\beta / 2)} .
$$

By the corollary to Lemma 4, (6.2) is equivalent to

$$
\sum_{\lambda=1}^{\infty} 2_{\omega}^{(1-\beta / 2) \lambda x \beta}\left(\delta 2^{-\lambda}\right)=\infty \text {, or } \sum_{\lambda} 2^{(1-\beta / 2) \lambda x}{ }_{\epsilon_{\lambda}}^{\beta}=\infty,
$$

hence $\sum \gamma_{N}^{\beta}=\infty$.

Next, in the same manner as in $\$ 5$, one can prove that

$$
M_{2}^{2}\left(u_{n}\right)=O \alpha_{n}^{2} ;
$$

it is easily seen that [5, formula (6.14)]

$$
\alpha_{n}^{2}=\left(\epsilon_{n}^{\beta}-\epsilon_{n+1}^{\beta}\right)^{2 / \beta}=O\left(\epsilon_{n}^{2}-\epsilon_{n+1}^{2}\right),
$$

hence

$$
M_{2}^{2}(G)=\sum_{n=0}^{\infty} M_{2}^{2}\left(u_{n}\right)=O \sum_{n}\left(\epsilon_{n}^{2}-\epsilon_{n+1}^{2}\right)=O(1) .
$$

Finally, to prove (6.3), write

$$
M_{2}^{2} \phi=\sum_{0}^{\infty} M_{2}^{2}\left\{u_{\nu}(X ; t)-u_{\nu}(X)\right\}=\sum_{0}^{n}+\sum_{n+1}^{\infty}=T_{1}+T_{2}
$$

say. From (6.6) and (6.7), $T_{2}=O \epsilon_{n}^{2}$, while, if we employ Lemma 9 (as in $\S 5$ )

$$
\begin{aligned}
T_{1} & =t^{2} O \sum_{0}^{n} 2^{2 \nu} \alpha_{\nu}^{2}=t^{2} O \sum_{0}^{n} 2^{2 \nu}\left(\epsilon_{\nu}^{2}-\epsilon_{\nu+1}^{2}\right) \\
& =t^{2} O \sum_{1}^{n} \epsilon_{\nu}^{2}\left(2^{2 \nu}-2^{2 v-2}\right)=t^{2} O \sum_{1}^{n}\left(2^{\nu}-2^{n-1}\right) 2^{n} \epsilon_{\nu}^{2} \\
& =t^{2} O \int_{1}^{2^{n}} x \omega^{2}\left(\delta x^{-1}\right) d x=t O \int_{1}^{\delta-12^{n}} y \omega^{2}\left(y^{-1}\right) d y .
\end{aligned}
$$


Employing (6.1), we now get

$$
T_{1}=t^{2} O 2^{2 n} \omega^{2}\left(\delta 2^{-n}\right) .
$$

Given a positive $t$, choose $n$ so that

$$
2^{n}<\delta / t \leqq 2^{n+1} ;
$$

then

$$
T_{1}=t^{2} O t^{2} \omega^{2}(t)=O \omega^{2}(t), \text { and } T_{2}=O \omega^{2}(t)
$$

hence

$$
M_{2} \phi(t)=O \omega(t) \quad \text { as } t \rightarrow 0 .
$$

This proves Theorem 5 . 6.1]).

Remark. The conditions (5.2) and (6.1) are equivalent (cf. [5, Remark

7. A continuous function as counter example. In $[5, \S 6]$ we have employed polynomials

$$
g(z)=\sum_{v=0}^{2(q-1)} a_{p}^{(q)} z_{;}^{\prime}, \quad q \text { a prime } \equiv 1(\bmod 4),
$$

with the following properties

$$
\begin{array}{lr}
|g(z)| \leqq 1 & \text { for }|z| \leqq 1, \\
\left|a_{\nu}^{(q)}\right|=q^{-8 / 2}(\nu+1), & \nu=0,1, \cdots, q-2 .
\end{array}
$$

On putting $g\left(z_{1}\right) \cdots g\left(z_{\kappa}\right)=\sum b_{N} z_{1}^{n_{1}} \cdots z_{\kappa}^{n_{\kappa}}$, it follows that

$$
\begin{aligned}
\sum\left|b_{N}\right|^{\beta}>\left(\sum\left|a_{v}^{(q)}\right|^{\beta}\right)^{\alpha} & >q^{-3 \beta \kappa / 2}\left(1^{\beta}+2^{\beta}+\cdots+(q-1)^{\beta}\right)^{k} \\
& >\frac{1}{\kappa+1} q^{-3 \beta \kappa / 2}(q-1)^{\alpha(\beta+1)} .
\end{aligned}
$$

Let $1<q_{1}<q_{2}<\cdots$ be a sequence of primes congruent to $1(\bmod 4)$, and such that for all large $n$

$$
2^{n-1}<q_{n}<2^{n}
$$

denote by $g_{n}(z)$ the polynomial (7.1) with $q=q_{n}$, and let

$$
\lambda_{1}=0, \quad \lambda_{n+1}=2\left(q_{1}+\cdots+q_{n}\right)-n,
$$$$
n \geqq 1 ;
$$

$\epsilon_{n}, \alpha_{n}$, and $u_{n}$ are defined as in $\S 6$. We assume that $\omega(t)$ satisfies the conditions of Theorem 5 and, in case $1<\beta<2$, the additional conditions

$$
\int_{1}^{\infty} x^{-1} \omega\left(x^{-1}\right) d x=\int_{0}^{1} \tau^{-1} \omega(\tau) d \tau<\infty,
$$




$$
\int_{t-1}^{\infty} x^{-1} \omega\left(x^{-1}\right) d x=\int_{0}^{t} \tau^{-1} \omega(\tau) d \tau=O \omega(t)
$$

as $t \rightarrow 0$.

Now, as shown in $[5, \S 6]$,

$$
\sum_{1}^{n} 2^{n} \alpha_{\nu}<2 \int_{1}^{2^{n}} \omega\left(x^{-1}\right) d x
$$

and

$$
\sum_{n+1}^{\infty} \alpha_{\nu}< \begin{cases}\epsilon_{n+1} & \text { for } 0<\beta \leqq 1 \\ 2 \int_{2^{n}}^{\infty} x^{-1} \omega\left(x^{-1}\right) d x & \text { for } 1<\beta<2 .\end{cases}
$$

We define as before

$$
G(Z)=\sum_{1}^{\infty} u_{n}(Z)=\sum \gamma_{N} z_{1}^{n_{1}} \cdots z_{\kappa}^{n_{k}} .
$$

By (7.1)

$$
\left|u_{n}(Z)\right| \leqq \alpha_{n} \quad \text { for }\left|z_{1}\right| \leqq 1, \cdots,\left|z_{\alpha}\right| \leqq 1,
$$

hence the simple series in (7.9) converges uniformly and defines a continuous function in $\left|z_{1}\right| \leqq 1, \cdots,\left|z_{k}\right| \leqq 1$. Putting $z_{\nu}=\exp \left(i x_{\nu}\right), \nu=1, \cdots, \kappa,(7.9)$ becomes the Fourier power series of a continuous function $F\left(x_{1}, \cdots, x_{k}\right)$. Furthermore, using (7.2) and (7.3), we have

$$
\begin{aligned}
\sum\left|\gamma_{N}\right|^{\beta} & >\frac{1}{\kappa+1} \sum_{n} \alpha_{n}^{\beta} q_{n}^{-3 \kappa \beta / 2}\left(q_{n}-1\right)^{\kappa(\beta+1)} \\
& >b \sum_{n}\left(\epsilon_{n}^{\beta}-\stackrel{\beta}{\epsilon}+1\right)^{\beta \kappa(1-\beta / 2)}
\end{aligned}
$$

b a constant,

and the divergence of this series follows from (6.2) as in $\$ 6$.

We shall finally show that the modulus of continuity of $F(X)$ is majorized by $\omega(t)$. We define the modulus of continuity of $F(X)$ by

$$
\max _{|H| \leqq t} \max _{(X)}|F(X+H)-F(X)|=\zeta(t),
$$

where $|H|=\left(h_{1}^{2}+\cdots+h_{\kappa}^{2}\right)^{1 / 2}$, and each $x_{v}$ varies in $(-\pi, \pi)$. Now, in view of (7.9),

$$
\begin{aligned}
|F(X+H)-F(X)| & \leqq \sum_{1}^{\infty}\left|u_{r}\left(e^{i\left(x_{1}+h_{1}\right)}, \cdots, e^{i\left(x_{n}+h_{k}\right)}\right)-u_{\nu}\left(e^{i x_{1}}, \cdots\right)\right| \\
& =\sum_{1}^{n}+\sum_{n+1}^{\infty}=V_{1}+V_{2},
\end{aligned}
$$


say. From (7.10) and (7.8)

$$
V_{2}<2 \sum_{n+1}^{\infty} \alpha_{\nu}< \begin{cases}2 \epsilon_{n} & \text { for } 0<\beta \leqq 1, \\ 2 \int_{2^{n}}^{\infty} x^{-1} \omega\left(x^{-1}\right) d x & \text { for } 1<\beta<2 ;\end{cases}
$$

in view of (7.6) we have in either case

$$
V_{2}=O \omega\left(\delta 2^{-n}\right) .
$$

To estimate $V_{1}$, we employ as in $\$ 5$ the mean value theorem, and Lemma 9 for $p=\infty$. We then get

$$
V_{1}<\left(\sum_{1}^{k}\left|h_{\nu}\right|\right)\left(\sum_{1}^{n} \alpha_{\nu} \lambda_{\nu+1}\right)
$$

and, using (7.7) and (5.2) (which is equivalent to (6.1)),

$$
V_{1}=O|H| 2^{n} \omega\left(\delta 2^{-n}\right) \text {. }
$$

For $|H| \leqq t$ choose $n$ so that $2^{n-1}<t^{-1} \leqq 2^{n}$, then

$$
V_{1}=O \omega(t) \text { and } V_{2}=O \omega(t)
$$

hence

$$
\zeta(t)=O \omega(t) .
$$

We have thus proved the theorem:

THEOREM 6. If the assumptions of Theorem 5 are satisfied and if $0<\beta \leqq 1$, then there exists a continuous function $F(X)$ with modulus of continuity $\zeta(t)<\omega(t)$, while $\sum\left|c_{N}\right|^{\beta}=\infty$. The same result holds for $1<\beta<2$ under the additional assumptions (7.5) and (7.6).

As an example choose $\omega(t)=t^{\alpha}, 0<\alpha<i$; it is seen easily that now (6.1), (7.5), and (7.6) hold. Theorem $1^{\prime \prime}$ yields the convergence of $\sum\left|c_{N}\right|^{\beta}$ whenever $M_{2} \phi=O t^{\alpha}$, and if $\beta>2 \kappa /(\kappa+2 \alpha)$. For $\beta=2 \kappa /(\kappa+2 \alpha)$, however, there exists a continuous function whose modulus of continuity is less than $t^{\alpha}$, while $\sum\left|c_{N}\right|^{\beta}=\infty$.

Closing remark. In a similar manner the convergence of the series $\sum|N|^{\alpha}\left|c_{N}\right|^{\beta}$ can be discussed. The mode of procedure applies as well to Fourier integrals. We may also consider instead of the spherical mean (2.14) the more general average

$$
f_{p}(X ; t)=\frac{c_{p}}{t^{k}} \int_{0}^{t}\left(1-\frac{r^{2}}{t^{2}}\right)^{p-1} f(X ; r) r^{\alpha-1} d r .
$$

Finally, if we denote the linear operator which transforms $f(X)$ into $f(X ; t)$ $-f(X)$ by $\Delta f(X ; t)$, iteration yields 


$$
\Delta_{m} f(X ; t) \sim \sum c_{N}\left(\alpha_{\mu}(t|N|)-1\right)^{m} \exp (i N X), \quad m=1,2,3, \cdots,
$$

and in Theorem 1 the assumption $M_{p} \phi(X ; t) \equiv M_{p} \Delta_{1} f(X ; t)=O \omega(t)$ can be replaced by $M_{p} \Delta_{m} f(X ; t)=O \omega(t)$.

\section{REFERENCES}

1. S. Bochner, Summation of multiple Fourier series by spherical means, Trans. Amer. Math. Soc. vol. 40 (1936) pp. 175-207.

2. H. Bohr and H. Cramér, Encyklopädie der Mathematischen Wissenschaften, vol. II, Part 3, No. 8.

3. G. E. Reves and O. Szász, Some theorems on double trigonometric series, Duke Math. J. vol. 9 (1942) pp. 693-705.

4. F. Riesz, Uber eine Verallgemeinerung der Parsevalschen Formel, Math. Zeit. vol. 18 (1923) pp. 117-124.

5. O. Szász, Fourier series and mean moduli of continuity, Trans. Amer. Math. Soc. vol. 42 (1937) pp. 366-395.

6. G. N. Watson, Theory of Bessel functions, $2 \mathrm{~d}$ ed., 1944.

ANDHRA UNIVERSITY, WALTAIR, INDIA.

UNiversity OF CincinNati, Cincinnati, Оhio. 